\title{
Improving Location Algorithm based on Fuzzy Cluster in WSN
}

\author{
Xing Fang, Zhihong Qian, Chuxi Yang, Liang Qian \\ College of Communication Engineering, Jilin University, Changchun, Jilin 130012, China \\ E-mail:dr.qzh@163.com
}

\begin{abstract}
To reduce the impact of distance information errors, an improved location algorithm in WSN based on fuzzy clustering is proposed. It is explored that clustering methodology is employed to wireless sensor network localization schemes. By cluster analysis, the novel algorithm can figure out the distance data which are far more beyond their true value, and then removes those data from the measured distance data. Then multilateration is adopted with the rest distance data, obtaining the final results. Simulation experiments indicate that the improved algorithm can reduce location errors effectively and has more stable location results in a variety of error environment comparing with the original multilateration algorithm.
\end{abstract}

Keywords—wsn; multilateration; fuzzy cluster; location

\section{INTRODUCTION}

Location information is an important source of context for ubiquitous computing system. After entering the Inter of things era, the $80 \%$ of the information in IOT is concerned with position, the relative position between things is the basis of implementing things connected. The basis of the target location problem in the IOT has become one of research hot spots $^{[1,2]}$.

Multilateral positioning method is the basic algorithm which is widely used in wireless location. This method requires to obtain at least three distance information between the nodes to be located and anchor nodes. The distance information can be obtained by ranging technology such as TOA 、 RSSI, also can be estimated by DV-Hop 、 DVdistance $^{[3,4]}$. However, in practical applications, due to various factors such as hardware、 environment、propagation model, measured or estimated distance often has some errors ${ }^{[5]}$. And NLOS cause a greater impact on the positioning accuracy ${ }^{[6]}$. The distance deviated from the true will affect the positioning accuracy.

In the case of the impact of ranging error of multilateral localization algorithm ${ }^{[7]}$, the researchers have also done a lot of work, focusing mainly on two aspects. One is from the perspective of solving equations, using different methods for the optimal solution of nonlinear equations, such as Tikhonov regularization method, the steepest descent method, Landweber iterative method; On the other hand, it is to give weights on ranging information based on certain principles associated with error message, reducing the influence of big error ranging positioning results.

Overall, the above methods are studied separately for the ranging error and positioning solution method, which mainly study how to reduce the impact of ranging error on positioning results. But for measurements with larger errors, the above method can not completely remove the impact on the results of the positioning. Therefore, we propose multilateral localization algorithm based K-means clustering. Cluster analysis excludes some large error distance information, solving error distance affect the positioning results.

\section{Multilateration LOCATION AlgORITHM}

In wireless sensor network positioning, position known nodes are called anchor nodes and to be positioned, position unknown node are called unknown nodes. Multilateral localization algorithm obtains positioning results by obtaining the distance between unknown nodes and anchor nodes and establishing distance equations ${ }^{[8,9]}$.

Suppose the unknown node $U$ real coordinates $\left(x_{0}, y_{0}\right)$, solutions of variable node $U$ is represented by coordinates $(x, y)$; anchor nodes $M_{1}, M_{2}, \cdots \cdots, M_{n}$ positions are known: $\left(x_{1}, y_{1}\right),\left(x_{2}, y_{2}\right), \cdots \cdots,\left(x_{n} y_{n}\right)$; the distances between unknown node and anchor nodes $M_{1}, M_{2}, \cdots \cdots, M_{n}$ are $l_{1}, l_{2}, \cdots \cdots, l_{n}$; node $U$ has achieved the distance between $M_{1}, M_{2}, \cdots \cdots, M_{n}$ : $d_{1}, d_{2}, \cdots \cdots, d_{n}$ 。 According to the two-dimensional plane geometry:

$$
\left\{\begin{array}{l}
\left(x-x_{1}\right)^{2}+\left(y-y_{1}\right)^{2}=d_{1}^{2} \\
\left(x-x_{2}\right)^{2}+\left(y-y_{2}\right)^{2}=d_{2}^{2} \\
\left(x-x_{3}\right)^{2}+\left(y-y_{3}\right)^{2}=d_{3}^{2} \\
\cdots \cdots \\
\left(x-x_{n}\right)^{2}+\left(y-y_{n}\right)^{2}=d_{n}^{2}
\end{array}\right.
$$

Coordinates $(x, y)$ of equation (1) is the unknown node.

In equations, the 1 to $(n-1)$ equation minus the $n$ equation respectively, and seek the optimal solution. It can be obtained unknown node coordinate:

$$
\hat{X}=\left(A^{T} A\right)^{-1} A^{T} B
$$

Among them, 


$$
\begin{gathered}
A=\left[\begin{array}{ll}
2\left(x_{n}-x_{1}\right) & 2\left(y_{n}-y_{1}\right) \\
\cdot & \cdot \\
\cdot & \cdot \\
2\left(x_{n}-x_{n-1}\right) & 2\left(y_{n}-y_{n-1}\right)
\end{array}\right], \quad X=\left[\begin{array}{l}
x \\
y
\end{array}\right], \\
B=\left[\begin{array}{l}
d_{1}^{2}-d_{n}^{2}+y_{n}^{2}+x_{n}^{2}-y_{1}^{2}-x_{1}^{2} \\
d_{2}^{2}-d_{n}^{2}+y_{n}^{2}+x_{n}^{2}-y_{2}^{2}-x_{2}^{2} \\
\cdot \\
\cdot \\
d_{n-1}^{2}-d_{n}^{2}+y_{n}^{2}+x_{n}^{2}-y_{n-1}^{2}-x_{n-1}^{2}
\end{array}\right]
\end{gathered}
$$

Since the measured or estimated distance $d_{1}, d_{2}, \cdots \cdots, d_{n}$ and the distance between the unknown node and anchor nodes $l_{1}, l_{2}, \cdots \cdots, l_{n}$ has the error,

$$
r_{i}=\left|l_{i}-d_{i}\right| / l_{i}, \quad(i=1,2, \ldots \ldots, n)
$$

Thus the position by using multilateral positioning method $(x, y)$ and the real unknown node $\left(x_{0}, y_{0}\right)$ has certain error, denoted by

$$
R M S E=\sqrt{\left(x-x_{0}\right)^{2}+\left(y-y_{0}\right)^{2}}
$$

\section{IMPROVING LOCATION ALGORITHM BASED ON FUZZY CLUSTER}

Using three distance information to location in twodimensional space, that is trilateral measurement method. In the presence of the ranging error, the distance information which has a small ranging error has less little effect on the trilateral, thus positioning results are closer to the true value. In the case of some distance information relatively accurate, the obtained results using the trilateral measurement method by three distance information will be more close to the real value, which densely distributed in an unknown true value of the node position as the center of a region.

Based on this, we propose improving location algorithm based on fuzzy cluster. It randomly selects 3 from $n$ distance information, and obtains sample collections using trilateral measurement method. By cluster analysis, the novel algorithm can figure out the distance data which are far more beyond their true value, and then removes those data from the measured distance data. Then multilateration is adopted with the rest distance data, obtaining the final results.

Firstly, improving location algorithm based on fuzzy cluster selects a group of 3 from $n$ distance information $\left\{d_{1}, d_{2}, \cdots \cdots, d_{n}\right\}$. It obtains $C_{n}^{3}$ positioning results $\left(X=\left\{\left(x_{i}, y_{i}\right) \mid i=1,2, \cdots \cdots, C_{n}^{3}\right\}\right) \quad$ using trilateral measurement method to every group. Then it filters distance information of big error and do k-means cluster in sample points $\circ C_{n}^{3}$ sample points are divided into $\mathrm{k}$ clusters, as shown in step A; Then according to the result of clustering, it performs step $B$ to find distance information of big error.

- Step A k-means cluster of initial sample
1) Input $C_{n}{ }^{3}$ sample to be cluster $X=\left\{\left(x_{i}, y_{i}\right) \mid i=1,2, \cdots \cdots, C_{n}^{3}\right\}$ and cluster number k. Choose the number of $\mathrm{k}$ points from the sample of $C_{n}{ }^{3}$ as the initial clustering center.

2) For the rest of $\left(C_{n}^{3}-k\right)$ points, it calculates the distance $D=\left\{d_{i j} \mid i=1,2, \cdots \cdots,\left(C_{n}^{3}-k\right) ; j=1,2, \cdots k\right\}$ between each sample and the center of each cluster $\left(c_{i x}, c_{i y}\right) . D_{i j}$ denotes the distance between the point $\mathrm{i}$ and cluster center $\mathrm{j}$. Find the nearest cluster center $j(1 \leqslant j \leqslant k)$ to the point $i$.

3) All points join clustering and form the $\mathrm{k}$ cluster $W_{1}, W_{2}, \cdots \cdots, W_{k}$, each clustering $W_{i}$ includes clustering center $C_{i}$ 、 the points belong to the cluster and the number of cluster points.

4) According to the formula $c_{i x}{ }^{*}=\left(1 / n_{i}\right) \sum x, \quad c_{i y}{ }^{*}=\left(1 / n_{i}\right)$ $\Sigma y$, it computes center of each cluster, until find new cluster center $C^{*}=\left\{\left(c_{i x}{ }^{*}, c_{i y}{ }^{*}\right) \mid i=1,2, \cdots \cdots, k\right\} . x, y \in W_{i}, n_{i}$ denotes the number of clustering $W_{i}$.

5) If $C^{*}=C$, that is, no change of two adjacent clustering center, end of the algorithm, at this time $W_{1}, W_{2}, \cdots \cdots, W_{k}$ are ultimately clusters. Otherwise make $C^{*}=C$, turn to step 2 .

- Step B To find the distance information of big error according to the cluster results

1) According to the final output of the K-means clustering algorithm $W_{1}, W_{2}, \cdots \cdots, W_{k}$ and $n_{1}, n_{2}, \cdots \cdots, n_{k}$, it finds $t(2 \leqslant t \leqslant k-1)$ clusters of minimum number of points.

2) To find out points be contained by $t$ clusters, supposing $h$ points. Each point contains 3 distance information. Then we can find the top number of $m$ distance information, supposing they are $d_{1}, d_{2}, \cdots \cdots, d_{m}$. This are distance of big error.

\section{SimUlation}

All the simulations are conducted under such a model that 15 anchor nodes are deployed with uniform distribution in an object field with $100 \mathrm{~m} \times 100 \mathrm{~m}$ square meters. The to-belocated node is standing in the center of this field with coordinate $(50,50)$. We experiment between Multilateration and improved algorithm. Each group of positioning error of the experimental results are the average of 100 times of experiments. The parameters of improved algorithm set $k=5$, $t=2$. In the simulation experiment of the improved algorithm $m$ take 1,2 and 3 respectively. The experimental results with distance measurement error between the unknown node and anchor node which in normal distribute and uniform distribution are shown in figure 1 and 2 respectively. 


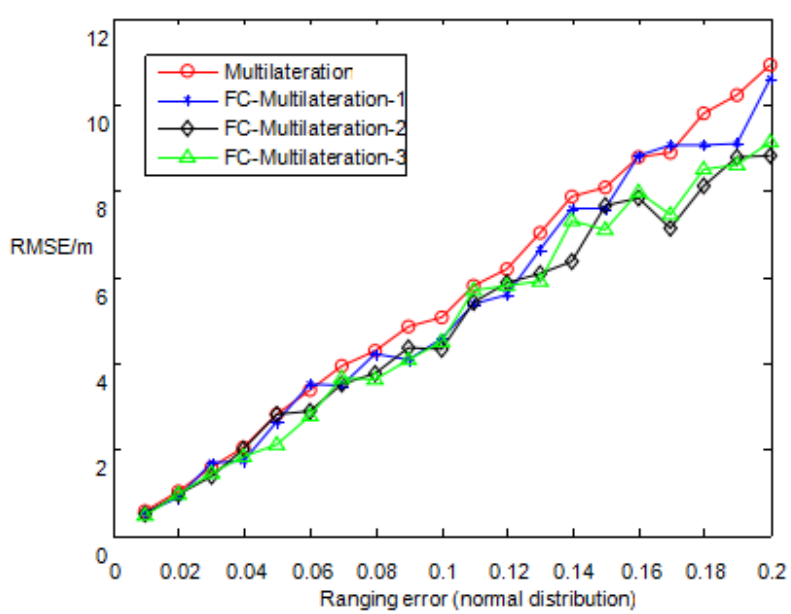

Fig.1 comparison of location error in the ranging error of normal distribution

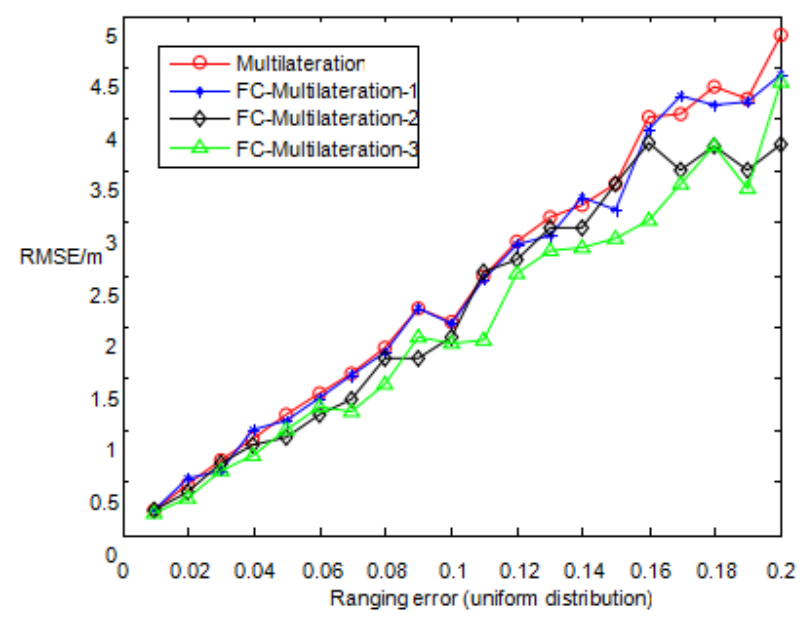

Fig. 2 comparison of location error in the ranging error of unifrom distribution

Fig. 1、2 show that even in the absence of a significantly larger measurement error value condition, no matter the ranging error obeys normal distribution or uniform distribution, the improved algorithm has smaller positioning error.

\section{CONCLUSION}

In the view of the influence of error ranging information contained on the multilateral localization algorithm, an improved location algorithm in WSN based on fuzzy clustering is proposed. It is explored that clustering methodology is employed to wireless sensor network localization schemes. By cluster analysis, the novel algorithm can figure out the distance data which are far more beyond their true value, and then removes those data from the measured distance data. Simulations show that the improved algorithm can effectively avoid the influence of error ranging information, and the improved algorithm has a good localization robustness to the multilateral positioning algorithm.

\section{ACKNOWLEDGMENT}

This work is supported by National Science Foundation of China (No.61371092).

\section{REFERENCES}

[1] QIAN Zhi-hong and WANG Yi-jun. Internet of things-oriented wireless sensor networks review[J]. Journal of Electronics \& Information Technology, 2013, 35(1): 215-227.

[2] QIAN Zhi-hong and WANG Yi-jun. IOT technology and application[J]. Acta Electronica Sinica, 2012, 40(5): 1023-1029.

[3] D. Niculescu, B. Nath. Ad Hoc Positioning System (APS)[C]. Proc. of the IEEE GLOBECOM 2001, San Antonio, 2001. 2926-2931.

[4] Liu Ying, Qian Zhihong, Liu Dan, Zhong Hui. A DV-Hop Positioning Algorithm for Wireless Sensor Network Based on Detection Probability [C]. 2009 5th International Joint Conference on INC, IMC and IDC (NCM 2009). 2009: 453-456.

[5] Horacio A.B.F. Oliveira, Eduardo F.Nakamura, Antonio A.F.Loureiro, Azzedine Boukerche. Error Analysis of Localization Systems for Sensor Networks[C]. In: Proc. of the 13th Annual ACM international workshop on Geographic Information Systems(GIS'05), Bermen, Germany, Nov, 2005 .

[6] Wanming Chen, Tao Mei, Lei Sun, et al.. Error Analyzing for RSSIbased Localization in Wireless Sensor Networks[C]. Proceedings of the 7th World Congress on Intelligent Control and Automation, Chongqing, China, June, 2008.

[7] Savarese C, Langendoen K, Rabaey J. Robust positioning algorithms for distributed ad-hoc wireless sensor networks[C]. USENIX Annual Technical Conference, June,2002. 317-327.

[8] Savvides A, Han C C, Srivastava M. Dynamic Fine-grained Localization in Ad-hoc Nerworks of Sensors[A]. Proceedings of the 7th Annual International Conference on Mobile Computing and Networking[C]. Rome: ACM Press,2001. 166-179.

[9] Kim Sunwoo, Lee Byeong-Tae. Scalable DV-Hop Localization Algorithm with Constrained Multilateration for Wireless Sensor Nerwork[J]. IEICE Transactions on Communications, 2009, E92.B(10): 3075-3078. 